\title{
Recent Advancement of Environmental TEM for Material Process Characterization
}

\author{
Seiji Takeda', Hideto Yoshida' ${ }^{1}$ and Tetsuya Uchiyama'
}

1. Institute of Scientific and Industrial Research, Osaka University, 8-1 Mihogaoka, Ibaraki, 567-0047 Osaka, Japan

Recent technological advancements in TEM such as aberration correction of lenses and fast detection cameras have drastically boosted the capabilities of in-situ environmental transmission electron microscopy (E-TEM) for various types of materials characterization. Two major capabilities are: the characterization of material synthesis processes, and the characterization of functional materials and devices at operating conditions. On the former, a miniaturized reaction chamber reproduces the material synthesis conditions inside an E-TEM apparatus and therefore allows us to obtain atomic scale information throughout the processing time. On the latter, we can characterize the atomic scale of catalysts, batteries and electronic devices, in real environments, by combining the E-TEM apparatus with simultaneous optical, electronic, and/or thermal add-on equipment. Therefore, in-situ E-TEM has greatly extended the fields applicable by TEM. The purpose of this presentation is to show our recent contribution to E-TEM on the aforementioned aspects.

Carbon nanotubes (CNTs) have regained great attraction for future electronic devices, partially because it has been recently shown that even the chirality of CNTs can be controlled during the dedicated synthesis processes. To show the capabilities of ETEM in characterizing synthesis processes, we reinvestigated the chemical vapor deposition (CVD) growth of CNTs, especially in Co-catalyzed CVD growth. In this type of CVD growth, gas-phase carbon is converted to CNTs, and solid carbon, on the surface and the inside of nanoparticulate catalysts. According to previous studies, the surface of crystalline Ni acts as the catalyst in Ni-catalyzed CVD growth, while in Fe-catalyzed CVD growth, the nanoparticles of iron carbide act as the catalyst. These structural data on nanoparticulate catalysts can be accounted for by phase diagrams. We pursued the structural determination of nanoparticulate catalysts in Co-catalyzed CVD growth. Though Co carbide is not listed in the binary phase diagram of Co and C, Co carbide formed partially in a nanoparticle, acting as catalyst for the growth of CNTs [1]. This E-TEM study stimulates further experimental and theoretical studies on the time-evolved interfacial structures between catalysts and growing carbon nanostructures such as graphene as well as CNTs. To enhance the applicability of E-TEM to materials synthesis characterization, an apparatus for depositing metal clusters on a substrate was build in an E-TEM apparatus. Figure 1 shows the growth processes of gold nanoparticles on a carbon coated micro-grid at room temperature. This application opens up a sequential in-situ investigation from the preparation of catalysts followed by the nucleation and growth of materials from the catalysts.

The surface of solid catalysts in reaction environments has been extensively investigated toward the improvement of existing catalysts and development of novel catalysts. As a recent application in this aspect, Cs-corrected E-TEM captured the surface structure of platinum at the atomic scale in both oxidation and reduction gas environments. In $\mathrm{O}_{2}$, atomic layers of Pt oxides started forming on the preferential facets of Pt nanoparticles at the early stage; then, entire oxidization on the whole surface of $\mathrm{Pt}$ nanoparticles followed. The oxides were reduced promptly to Pt by adding a small amount of $\mathrm{CO}$ or $\mathrm{H}_{2} \mathrm{O}$ vapor to the dominant $\mathrm{O}_{2}$, as is shown in Fig. 2. However, electron irradiation during E-TEM observation most likely activated the gases (especially the moisture); and therefore was capable of promoting or suppressing the oxidation and reduction processes at room temperature [2]. Therefore, it 
becomes crucially important to develop an analysis methodology to derive time-evolved atomic structures in TEM images. The present study signalizes that a background phenomenon that is induced by electron irradiation needs to be subtracted from in-situ E-TEM data to aid in the interpretation of relevant microscopy data in material process characterization.

\section{References:}

[1] Y. Kohigashi, H. Yoshida, Y. Homma and S. Takeda, Appl. Phys. Lett. 105 (2014) 073108.

[2] H. Yoshida, H. Omote, S. Takeda, Nanoscale, 6 (2014) 13113.
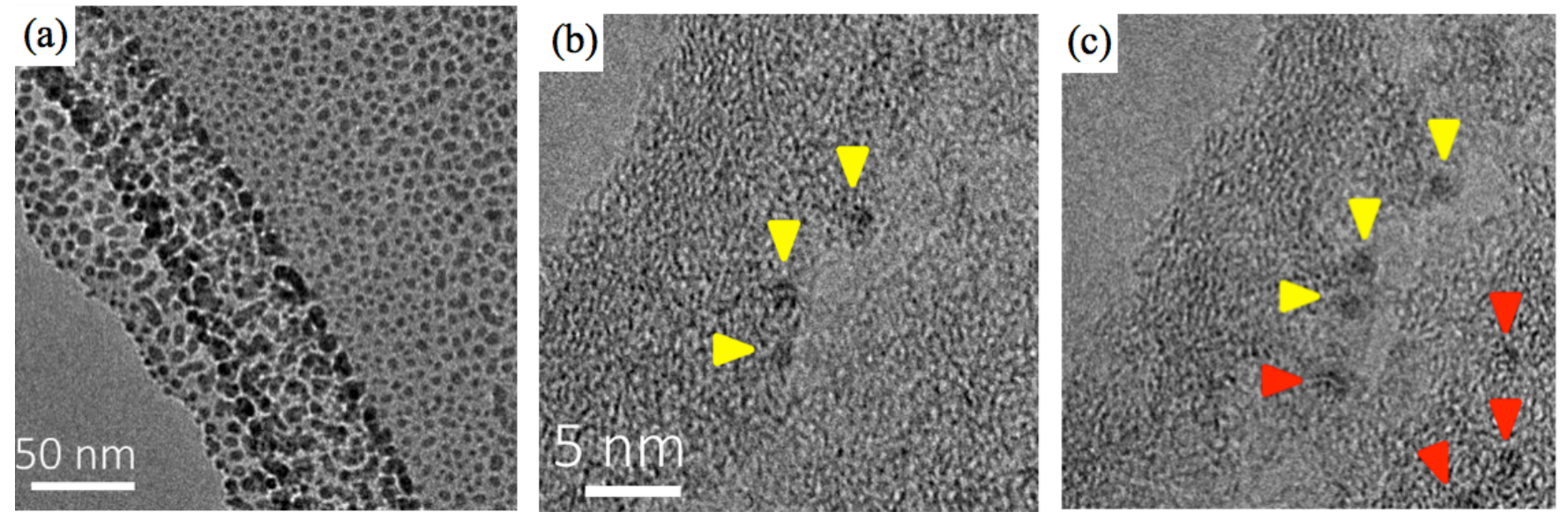

Figure 1. Depositing process of metal clusters on a substrate in an E-TEM apparatus at room temperature. (a) After deposition for about 300s at a certain deposition condition. (b) In-situ TEM in the similar deposition condition with deposition time from about $180 \mathrm{~s}$ in (b) to about 210s in (c). Metal nanoparticles grew as indicated by yellow arrow, while other nanoparticles nucleated as indicated by red arrows.
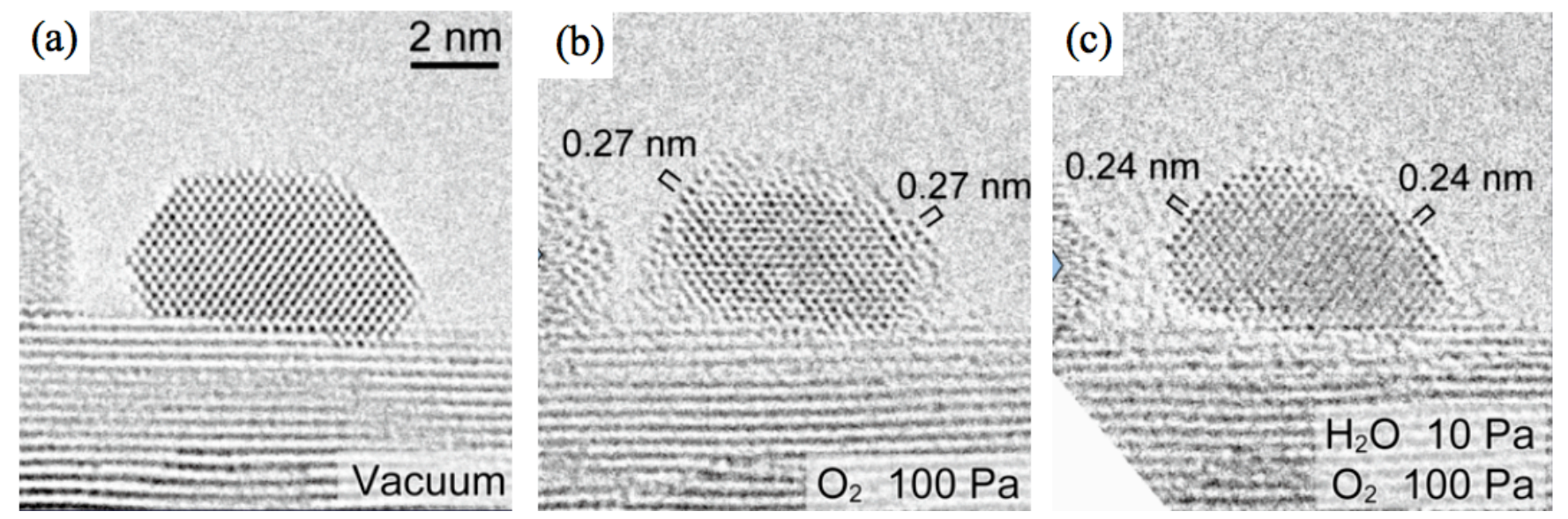

Figure 2. Reduction of metal oxide on the surface of platinum in $\mathrm{O}_{2}$ with moisture. (a) In vacuum as reference. (b) Formation of platinum oxide in $\mathrm{O}_{2}$ at room temperature. (c) Reduction of the oxide in $\mathrm{O}_{2}$ with moisture. Moisture $\left(\mathrm{H}_{2} \mathrm{O}\right)$ acted as a reducing agent. 